\title{
Low-energy elastic and inelastic scattering of positrons from argon
}

\author{
R. A. Boadle, T. J. Babij, J. R. Machacek, R. P. McEachran, and J. P. Sullivan* \\ Atomic and Molecular Physics Laboratories, Research School of Physics and Engineering, \\ Australian National University, Canberra, Australian Capital Territory 0200, Australia

\begin{abstract}
S. J. Buckman
Atomic and Molecular Physics Laboratories, Research School of Physics and Engineering, Australian National University, Canberra, Australian Capital Territory 0200, Australia and Institute of Mathematical Sciences, University of Malaya, Kuala Lumpur, Malaysia
\end{abstract}

(Received 7 October 2015; published 24 February 2016)

\begin{abstract}
Absolute measurements of elastic differential cross sections for positron scattering from argon at energies from $2-50 \mathrm{eV}$ are presented as well as total elastic and total inelastic cross sections from $2-20 \mathrm{eV}$. Comparisons of the current data, previous experimental data, and theoretical models using the convergent close-coupling and relativistic optical potential methods are made.
\end{abstract}

DOI: 10.1103/PhysRevA.93.022712

\section{INTRODUCTION}

Positron scattering from argon (Ar) at low energies is an ideal system in which to test quantum scattering theory. The noble gases, in general, are relatively simple targets for a theoretical treatment due to their closed valence shells and are available as high purity gases, making them experimentally easy to handle.

Absolute total scattering and positronium formation cross sections for argon have recently been measured [1] and the agreement between the experimental data and theory is generally good. Partial cross sections, such as the total elastic and total inelastic, give an additional level of information against which to test theory. By extending this, elastic differential cross sections (DCS) give the angular dependence of the elastic scattering and are more stringent tests for calculations.

The first experimental positron-Ar scattering DCS [2] were placed on an absolute scale using measured total scattering cross sections published in Ref. [3]. With one exception, other previous experimental DCS for positron-Ar scattering have been relative [4,5] and were typically scaled to match theoretical models at a specific angle, thus allowing comparison of the angular dependence only. Since these initial measurements, the development of the Surko buffer gas trap and highly sensitive manometers have enabled measurements of absolute DCS with improved angular resolution and statistical uncertainties. These absolute DCS provide information about both angular dependence and magnitude, but so far only a small number of energies have been studied using this technique [6]. The positron-Ar scattering system has also been studied theoretically, with the earliest predictions from the mid 1980s [7], quickly followed by further investigations such as Refs. [8,9]. This paper presents elastic differential cross sections for positron-Ar scattering at energies of 2 , $5,8,10,15,20,30$, and $50 \mathrm{eV}$ and includes total elastic cross sections from $2-20 \mathrm{eV}$ and total inelastic cross sections above the first electronic excitation threshold. New theoretical

\footnotetext{
*james.sullivan@anu.edu.au
}

calculations using the relativistic optical potential method applied to positron-Ar scattering are also presented.

\section{EXPERIMENTAL METHOD}

Experimental measurements were performed using the atomic and molecular positron beamline at the Australian National University. This apparatus and the associated experimental methods have been discussed in detail in previous publications [10-12], therefore it is only summarized here. Positrons are produced by decay of the radioactive source, sodium-22, and a solid neon moderator at $\sim 8 \mathrm{~K}$ provides a beam of positrons with an energy spread of $\sim 2 \mathrm{eV}$ full width at half maximum (FWHM). The moderated beam is guided using electric and magnetic fields, typically $530 \mathrm{G}$, into a Surko buffer gas trap. Here, the positrons are trapped and cooled by the combination of potentials provided by nine electrodes and collisions with nitrogen and tetrafluoromethane. They are subsequently released in an energy tunable, pulsed beam with an energy resolution of $\sim 60 \mathrm{meV}$ FWHM at a repetition rate of $\sim 50 \mathrm{~Hz}$. The pulsed beam is directed to the target $\mathrm{Ar}$ gas contained in a 5-cm-long, gold-plated copper scattering cell, with 5-mm-diameter entrance and exit apertures, at a pressure of $\sim 1$ mTorr. Postcollision, the positron parallel energy distribution $\left(E_{\|}\right)$, defined with respect to the direction of the magnetic field, is determined using a retarding potential analyzer (RPA), placed after the scattering cell. Combined with a microchannel plate detector the parallel energy intensity distribution, $I\left(E_{\|}\right)$, is measured. The relative potentials applied to the final electrode of the trap and the scattering cell allows the scattering energy of the positrons to be controlled.

In the case where only elastic scattering is possible, following a scattering event some of the positron's $E_{\|}$is transferred into the perpendicular component, $E_{\perp}$, due to scattering at some angle $\theta$. As the RPA is sensitive only to the parallel energy component, a measure of the induced $E_{\|}$ spread will correspond to a measurement of the scattering as a function of angle. The DCS is calculated [10,13] as

$$
\sigma_{\mathrm{DCS}}=-\frac{\sqrt{E E_{\|}}}{\pi n l} \frac{d I\left(E_{\|}\right)}{d E_{\|}},
$$


where $E$ is the scattering energy and $d I\left(E_{\|}\right) / d E_{\|}$is the slope of the parallel energy intensity distribution. The constant, $1 / n l$, where $n$ is the target number density and $l$ is the scattering path length (equivalent to the geometric length of the scattering cell), allows the measurement of absolute cross sections. DCS from this experiment actually represent cross sections which are "folded" about $90^{\circ}$. Positrons scattered at an angle $>90^{\circ}$ reflect off the final trap potential and traverse back through the scattering cell, meaning that the DCS at angle $\theta$ includes scattering at angle $180^{\circ}-\theta$ as well. As this backscattering means that some positrons make a second transit through the cell, multiple scattering events are a possibility and are mitigated by ensuring that the argon gas pressure is low enough so that fewer than $10 \%$ of the positrons scatter.

The total scattering cross section $\left(\sigma_{T}\right)$ is given by the BeerLambert law,

$$
\sigma_{T}=-\frac{1}{n l} \ln \left(\frac{I_{m}}{I_{o r}}\right),
$$

where $I_{m}$ is the transmitted positron intensity and $I_{o r}$ is the full, incident intensity. $I_{m}$ is measured at an offset, $\Delta V=90 \mathrm{meV}$ in this case, below the beam energy, such that scattered positrons can be distinguished from the unscattered beam, whereas $I_{o r}$ is the beam intensity when the scattering energy is below the positronium (Ps) formation threshold. The total elastic $\left(\sigma_{\text {elastic }}\right)$ and inelastic $\left(\sigma_{\text {inelastic }}\right)$ cross sections are calculated as fractions of $\sigma_{T}$. In order to calculate these, additional intensity measurements are made at the scattering energy, with the RPA at $0 \mathrm{~V}\left(I_{o}\right)$ and with the RPA at a potential corresponding to positron scattering at $90^{\circ}\left(I_{o}^{\prime}\right)$. The total elastic and total inelastic cross sections are thus

$$
\begin{aligned}
\sigma_{\text {elastic }} & =\frac{I_{o}^{\prime}-I_{m}}{I_{o r}-I_{m}} \sigma_{T}, \\
\sigma_{\text {inelastic }} & =\frac{I_{o}-I_{o}^{\prime}}{I_{o r}-I_{m}} \sigma_{T} .
\end{aligned}
$$

At scattering energies below the first electronic excitation threshold $(11.62 \mathrm{eV})$, the guiding magnetic field is kept at a constant value $(530 \mathrm{G})$ throughout the experiment. Above the first electronic excitation threshold, there is no longer a unique relationship between the $E_{\|}$distribution and the scattering angle, as energy can be lost due to inelastic scattering processes. In order to separate the elastic and inelastic scattering components, we can take advantage of the fact that the ratio between the perpendicular energy of a positron to the magnetic field strength $\left(E_{\perp} / B\right)$ is an adiabatic invariant if the magnetic field is slowly varying. Therefore, if the magnetic field in the RPA region $\left(B_{\mathrm{RPA}}\right)$ is reduced by a factor of $M=B_{\mathrm{SC}} / B_{\mathrm{RPA}}$, where $B_{\mathrm{SC}}$ is the magnetic field in the scattering cell region, the $E_{\|}$spread due to angular scattering is compressed, allowing those positrons that have been elastically scattered to be separated from those scattered inelastically [10]. The measurements presented here used $M=2$ for scattering energies from 11.62 to $20 \mathrm{eV}$ and $M=6$ for higher energies.

All scattering experiments are limited in their accessible angular range, and in most cases, this is defined by physical constraints, whereas here the limit is due to the energy resolution of the beam. The minimum angle, $\theta_{\min }$, which can be measured at a scattering energy, $E$, is given by

$$
\theta_{\min }=\sin ^{-1} \sqrt{\frac{e \Delta V}{E}},
$$

where $e$ is the positron charge. The inability to measure scattering below this minimum angle results in an underestimation of the total scattering cross section, as discussed in Refs. [14,15], and can be a significant effect. Corrections for the missing portion of the total elastic cross section are made using the methods outlined in Ref. [15].

Statistical uncertainties for the total elastic cross sections were $3-4 \%$, but higher for the total inelastic cross section $(>20 \%)$ and the DCS measurements $(>10 \%)$ dependent upon the energy or angle, respectively. Systematic uncertainties are estimated to be $\sim 3 \%$ and are mostly due to uncertainties in the number density of the gas based on pressure measurements made using a capacitance manometer (MKS Baratron Model 690A), with the largest contribution due to its zero drift.

\section{RESULTS AND DISCUSSION}

\section{A. Differential cross sections}

The DCS presented in Figs. 1 and 2 span an energy range from below the Ps formation threshold at $8.96 \mathrm{eV}$, where elastic scattering is the dominant scattering channel (direct annihilation is several orders of magnitude smaller and therefore considered negligible), to above the ionization threshold at $15.76 \mathrm{eV}$. The theoretical models shown are the convergent close-coupling (CCC) method [16] which includes electronic excitation and ionization, although it cannot separate direct ionization and ionization due to Ps formation, meaning that it can be inaccurate in the region between these thresholds. The static dipole polarizability calculated from the CCC theory is $13.7 a_{0}^{2}$, higher than the experimentally determined value of $11.08 a_{0}^{2}$ [17]; however, the total cross sections have been shown to agree well with experimental measurements [1]. The second theoretical comparison is the relativistic optical potential (ROP) [18] approach which forms a potential with real and imaginary parts attributed to the elastic and inelastic scattering processes, respectively. The static dipole polarizability is set to the experimental value of $11.08 a_{0}^{2}$, and above $8.96 \mathrm{eV}$ a simulation of Ps formation is incorporated [19] where Ps formation is modeled similarly to ioniszation, since both Ps formation and direct ionization leave the Ar atom in an ionized state. The present ROP theory uses the models developed in Refs. [18,19], applied to positron-Ar scattering.

Previous relative experimental data for positron-Ar DCS are taken from Floeder et al. [4] and Smith et al. [5], where the original data were scaled to match theory available at the time, while DCS drawn from Coleman and McNutt [2] were scaled using absolute total scattering cross-section measurements from Coleman et al. [3]. In order to compare with the present folded DCS, the shape of the unfolded CCC curve was used to estimate the effect of folding on the experimental data and the folded data were scaled to match the CCC calculation at $60^{\circ}$ for Floeder et al. [4] and Smith et al. [5]. The folded data from Coleman and McNutt [2] were rescaled, using total cross 

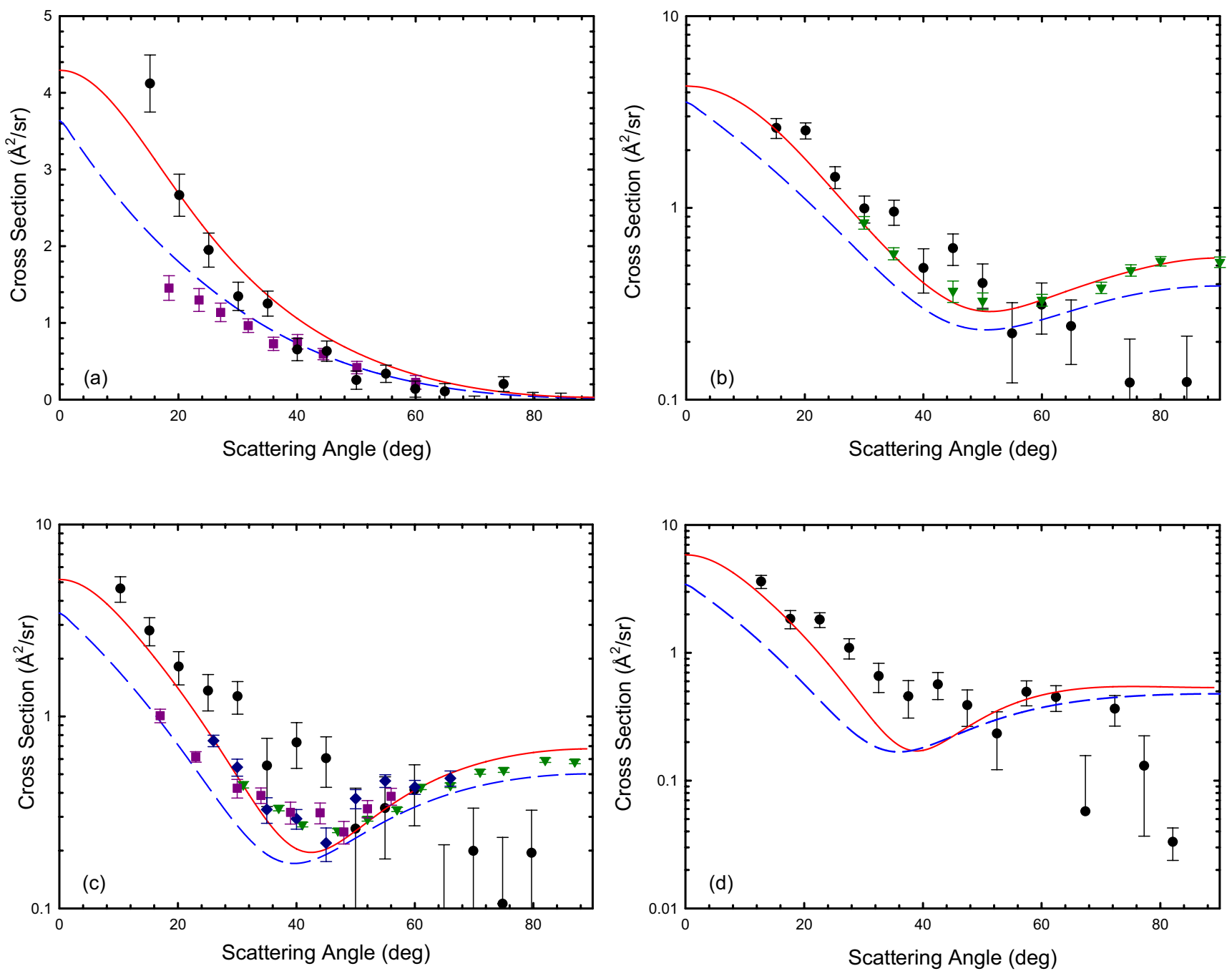

FIG. 1. Differential cross sections for positron scattering from $\mathrm{Ar}$ at (a) $2 \mathrm{eV}$, (b) $5 \mathrm{eV}$, (c) $8 \mathrm{eV}$, and (d) 10 eV. Previous experimental data are at the same scattering energy as the present results unless otherwise stated. $\bullet$, present data; - , CCC theory [16]; - - -, present ROP theory; $\checkmark$. Floeder et al. [at $8.5 \mathrm{eV}$ in panel (c)] [4]; $\mathbf{\nabla}$, Smith et al. [at $8.7 \mathrm{eV}$ in panel (c)] [5]; and $\mathbf{\square}$, Coleman and McNutt [at $2.2 \mathrm{eV}$ in panel (a) and at $8.7 \mathrm{eV}$ in panel (c)] [2]. Please note that, where the error bars are not plotted, the lower error bound extends below zero. In each case, the error bar is of a magnitude similar to that in the positive direction.

sections from Jones et al. [1], which we believe to give a more accurate value of the total, as demonstrated in Ref. [15].

Below the Ps formation threshold, the present data tend to agree better with the CCC theory, indicating a higher proportion of forward scattering than the ROP model, which generally underestimates the overall magnitude, consistent with the previous observations at the total cross-section level [1]. In particular, at $2 \mathrm{eV}$, the present data are excellently described by the CCC model at all angles, although the ROP is in good agreement at higher angles. The previous experimental data from Ref. [2], taken at $2.2 \mathrm{eV}$, are consistent with current data especially as the angle increases but demonstrates less forward angle scattering than is presently indicated.

Approaching the Ps formation threshold, both theoretical DCS at 5 and $8 \mathrm{eV}$ begin to predict a dip in the cross sections at $\sim 50^{\circ}$ and $\sim 40^{\circ}$ respectively, a trend which continues for the CCC theory above the threshold as shown in Figs. 1(d) and 2(a) -2 (c) for energies $10-30 \mathrm{eV}$. The dip is more dramatic at energies around the threshold and is echoed, especially, in the experimental data of Floeder et al. [4] taken at $8.5 \mathrm{eV}$ and Smith et al. [5] taken at 5 and $8.7 \mathrm{eV}$. In the 15 and $20 \mathrm{eV}$ data of Smith et al. [5] the dip is still present, but appears less distinct relative to the lower energy measurements. Smith et al. [5] attribute this effect to absorption due to the opening of the Ps channel. The current ROP theory, which includes the simulation of Ps formation that the CCC theory lacks, predicts no dips in the folded DCS above the Ps threshold and no similar features are observed in the present experimental DCS. However, there is a suggestion of a dip at $15 \mathrm{eV}$ in the present data, but large statistical uncertainties around $25^{\circ}$ to $30^{\circ}$ make this inconclusive. The reduction in the magnitude of the dip as additional scattering channels are modeled is consistent with previous theoretical work, such as Ref. [9] where the addition of electronic excitation reduced the dip by $\sim 60 \%$. However, overall the current data are consistent with previous experiments at all energies from $2-50 \mathrm{eV}$, with some small disagreement with Smith et al. [5] at higher scattering angles for 5 and $8 \mathrm{eV}$. At the higher energies from $15-50 \mathrm{eV}$, 

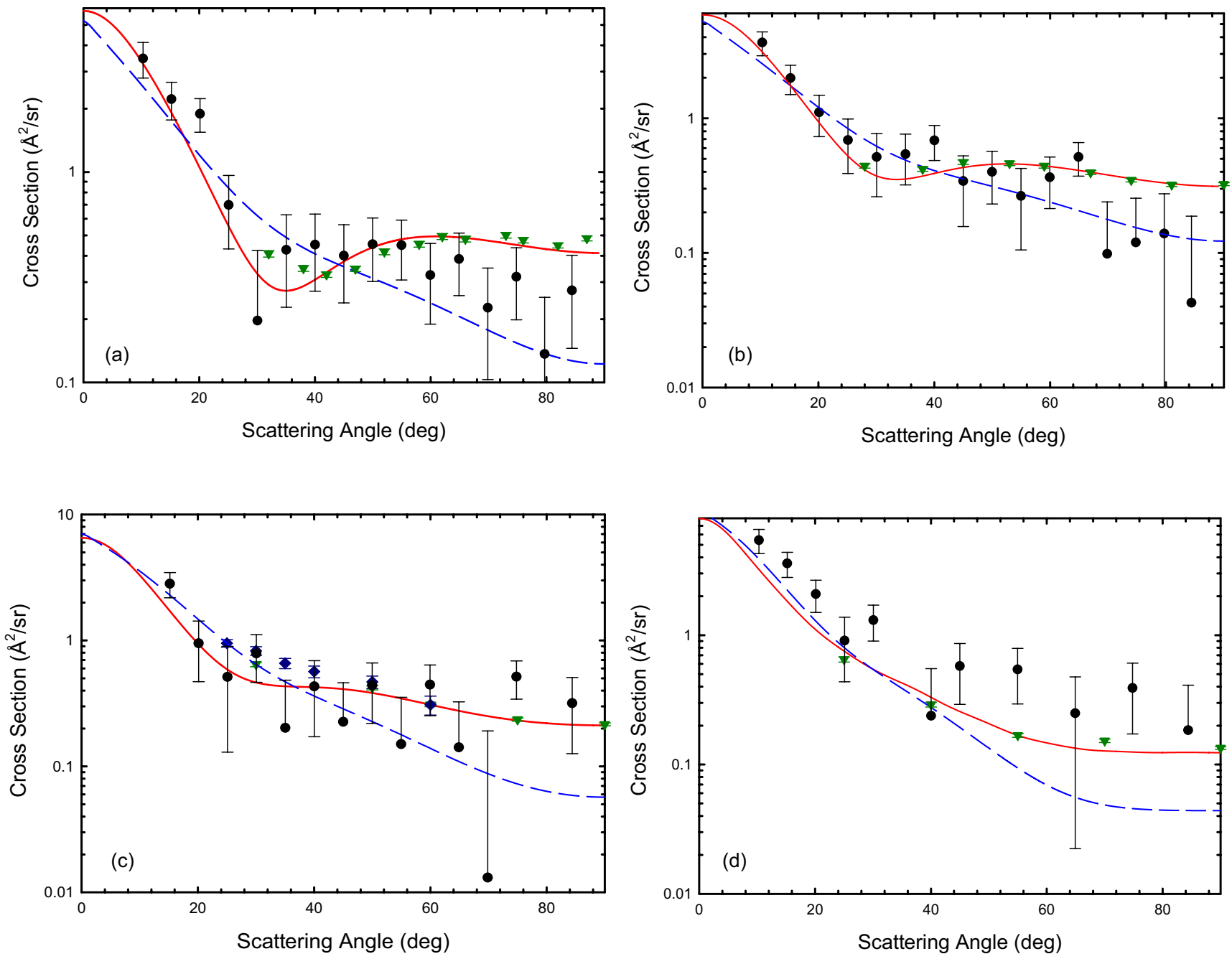

FIG. 2. Differential cross sections for positron scattering from Ar at (a) $15 \mathrm{eV}$, (b) $20 \mathrm{eV}$, (c) $30 \mathrm{eV}$, and (d) $50 \mathrm{eV}$. Previous experimental data are at the same scattering energy as the present results. $\bullet$, present data; - , CCC theory [16]; - - -, present ROP theory; $\downarrow$, Floeder et al. [4]; and $\mathbf{\nabla}$, Smith et al. [5]. Please note that where the error bars are not plotted, the lower error bound extends below zero. In each case, the error bar is of a magnitude similar to that in the positive direction.
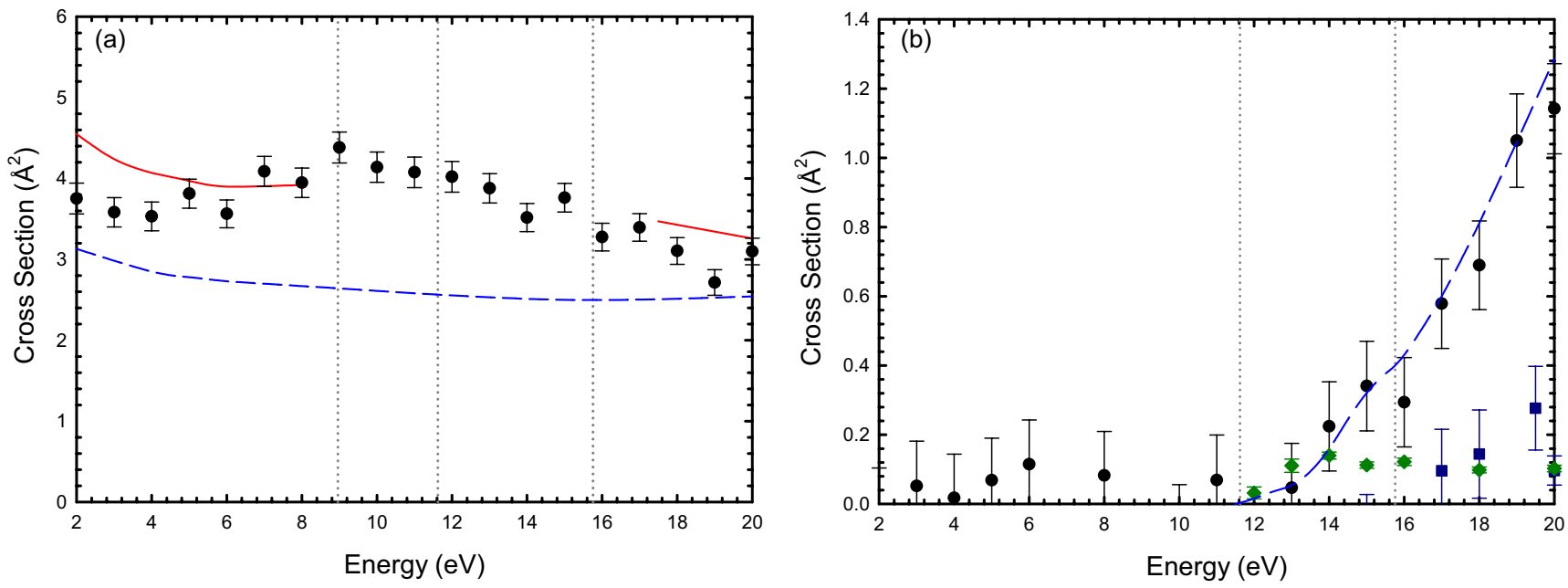

FIG. 3. (a) Total elastic scattering cross sections. Dotted vertical lines indicate the Ps formation threshold at $8.96 \mathrm{eV}$, the first electronic excitation threshold at $11.62 \mathrm{eV}$, and the ionization threshold at $15.76 \mathrm{eV}$. (b) Total inelastic scattering cross sections. Dotted vertical lines indicate the first electronic excitation threshold and the ionization threshold. $\bullet$, present data; - , CCC theory [16]; - - -, present ROP theory; $\bullet$, Sullivan et al. electronic excitation cross sections for the $3 p^{5}\left({ }^{2} P_{3 / 2,1 / 2}\right) 4 s$ states [20]; $\mathbf{\square}$, Marler et al. direct ionization cross sections [21]. 
TABLE I. Estimates of the total scattering cross-section corrections for selected energies with $\Delta V=90 \mathrm{meV}$ using ROP theory $[18,19]$.

\begin{tabular}{lcc}
\hline \hline Energy $(\mathrm{eV})$ & $\theta_{\min }\left({ }^{\circ}\right)$ & Correction $(\%)$ \\
\hline 2 & 17.2 & 22 \\
5 & 10.9 & 10 \\
15 & 6.3 & 7 \\
20 & 5.4 & 5 \\
\hline \hline
\end{tabular}

the present data are compatible with both theoretical models but no clear evidence of a dip is observed.

\section{B. Total elastic and inelastic cross sections}

The total elastic scattering cross section is shown in Fig. 3(a). The minimum angle [calculated using Eq. (5)] for these experiments leads to an underestimation of the total elastic and the total scattering cross section. Table I gives the minimum angles for selected energies and the corrections applied to the total elastic cross section which were determined using the shape of the ROP DCS curves (note that there is no significant difference when the CCC curves are used instead). Once these corrections have been performed, the present measurements are consistent with the total scattering cross sections of Ref. [1]. The total elastic cross sections are reasonably constant across the energy region from $2-20 \mathrm{eV}$ and the magnitude is generally well-modeled by the CCC theory. The CCC theory has difficulty converging in the region between the Ps formation threshold and the ionization threshold, therefore Fig. 3 does not display the CCC theory in this region. The ROP model predicts a smaller total elastic cross-section magnitude than measured, which is indicative of the discrepancies noted above between the ROP DCS forward angle scattering and the present experimental DCS.

Figure 3(b) shows the current measurements for the total inelastic cross section, including contributions from electronic excitation and ionization. Absolute cross sections for the $3 p^{5}\left({ }^{2} P_{3 / 2,1 / 2}\right) 4 s$ states [20] and direct ionization [21] are plotted for comparison. Below the ionization threshold, where only electronic excitation contributes to the present measurements, the current measurements are in reasonable agreement with those of Sullivan et al. [20]; however, at higher energies the present results are significantly higher than the sum of the measured electronic excitation and ionization cross sections. The excellent agreement between the experimental measurements and the ROP theory suggests that the large difference between the sum of data of Refs. [20] and [21] at $20 \mathrm{eV}$ can be explained by considering the contributions of the additional electronic excitations available at these energies as only the lowest electronic excitations were measured in the previous work. This agreement also indicates that the difference between the total scattering cross sections and the ROP theory discussed in Ref. [1] is most likely due to disagreements in the elastic component of the scattering process.

\section{CONCLUSION}

In this paper, absolute measurements have been presented of the elastic DCS from 2-50 eV positrons and of total elastic and total inelastic cross sections for positron scattering from Ar up to $20 \mathrm{eV}$.

At all energies, the present results are generally compatible with those of past experiments and tend to be in better agreement with the CCC model rather than the ROP method below the Ps formation threshold. However, at higher energies, where the ROP includes a model of Ps formation, the agreement between this theory and the measurements improves. Across the energy range, the dips in the DCS observed in some previous measurements, which were attributed to absorption effects, are not apparent in the current data.

Finally, the magnitude of the total elastic cross section agrees well with the CCC model and is higher than the ROP model, consistent with the discrepancies between the ROP method and experiment in the DCS forward angle scattering. The current total inelastic cross section also agrees reasonably with previous experimental measurements of the first electronic excitation cross sections, but by $20 \mathrm{eV}$ there is a large discrepancy between current data and past experiments. The ROP theory describes the total inelastic cross sections presented extremely well at all energies.

\section{ACKNOWLEDGMENTS}

The authors would like to acknowledge the Australian Research Council for funding support and one of the authors, R.A.Bo., would like to thank the Australian Government Department of Education and Training for the provision of an International Postgraduate Research Scholarship and an Australian Postgraduate Award. S.J.B. acknowledges the support of a visiting professorship from the University of Malaya. We are grateful to Igor Bray of Curtin University of Technology for provision of theoretical support and would like to thank our technical staff, Stephen Battisson and Ross Tranter, for their expertise and assistance.
[1] A. C. L. Jones, C. Makochekanwa, P. Caradonna, D. S. Slaughter, J. R. Machacek, R. P. McEachran, J. P. Sullivan, S. J. Buckman, A. D. Stauffer, I. Bray, and D. V. Fursa, Phys. Rev. A 83, 032701 (2011).

[2] P. G. Coleman and J. D. McNutt, Phys. Rev. Lett. 42, 1130 (1979).

[3] P. G. Coleman, J. D. McNutt, L. M. Diana, and J. T. Hutton, Phys. Rev. A 22, 2290 (1980).
[4] K. Floeder, P. Honer, W. Raith, A. Schwab, G. Sinapius, and G. Spicher, Phys. Rev. Lett. 60, 2363 (1988).

[5] S. J. Smith, G. M. A. Hyder, W. E. Kauppila, C. K. Kwan, and T. S. Stein, Phys. Rev. Lett. 64, 1227 (1990).

[6] S. J. Gilbert, R. G. Greaves, and C. M. Surko, Phys. Rev. Lett. 82, 5032 (1999).

[7] R. P. McEachran and A. D. Stauffer, in Positron (Electron)Gas Scattering, edited by W. E. Kauppila, T. S. Stein, 
and J. Wadehra (World Scientific, Singapore, 1985), pp. 122-130.

[8] H. Nakanishi and D. M. Schrader, Phys. Rev. A 34, 1823 (1986).

[9] K. Bartschat, R. P. McEachran, and A. D. Stauffer, J. Phys. B 21, 2789 (1988).

[10] J. P. Sullivan, S. J. Gilbert, J. P. Marler, R. G. Greaves, S. J. Buckman, and C. M. Surko, Phys. Rev. A 66, 042708 (2002).

[11] J. P. Sullivan, A. Jones, P. Caradonna, C. Makochekanwa, and S. J. Buckman, Rev. Sci. Instrum. 79, 113105 (2008).

[12] L. Chiari, E. Anderson, W. Tattersall, J. R. Machacek, P. Palihawadana, C. Makochekanwa, J. P. Sullivan, G. García, F. Blanco, R. P. McEachran, M. J. Brunger, and S. J. Buckman, J. Chem. Phys. 138, 074301 (2013).

[13] S. Gilbert, J. Sullivan, R. Greaves, and C. Surko, Nucl. Instrum. Methods Phys. Res., Sect. B 171, 81 (2000).
[14] M. Charlton and J. W. Humberston, Positron Physics (Cambridge University Press, Cambridge, England, 2000).

[15] J. P. Sullivan, C. Makochekanwa, A. Jones, P. Caradonna, D. S. Slaughter, J. Machacek, R. P. McEachran, D. W. Mueller, and S. J. Buckman, J. Phys. B 44, 035201 (2011).

[16] D. V. Fursa and I. Bray, New J. Phys. 14, 035002 (2012).

[17] R. R. Teachout and R. T. Pack, At. Data Nucl. Data Tables 3, 195 (1971).

[18] S. Chen, R. P. McEachran, and A. D. Stauffer, J. Phys. B 41, 025201 (2008).

[19] R. P. McEachran and A. D. Stauffer, J. Phys. B 46, 075203 (2013).

[20] J. P. Sullivan, J. P. Marler, S. J. Gilbert, S. J. Buckman, and C. M. Surko, Phys. Rev. Lett. 87, 073201 (2001).

[21] J. P. Marler, J. P. Sullivan, and C. M. Surko, Phys. Rev. A 71, 022701 (2005). 